\title{
La situation d'apprentissage : d'un lieu externe à un espace interne
}

Danielle Chini

\section{OpenEdition}

\section{Journals}

Édition électronique

URL : http://journals.openedition.org/asp/1507

DOI : 10.4000/asp. 1507

ISBN : 978-2-8218-0390-9

ISSN : 2108-6354

Éditeur

Groupe d'étude et de recherche en anglais de spécialité

Édition imprimée

Date de publication : 30 décembre 2002

Pagination : $95-108$

ISSN : 1246-8185

\section{Référence électronique}

Danielle Chini, « La situation d'apprentissage : d'un lieu externe à un espace interne », ASp [En ligne], 37-38 | 2002, mis en ligne le 24 juillet 2010, consulté le 01 mai 2019. URL : http://

journals.openedition.org/asp/1507 ; DOI : 10.4000/asp.1507

Ce document a été généré automatiquement le 1 mai 2019

Tous droits réservés 


\title{
La situation d'apprentissage : d'un lieu externe à un espace interne
}

\author{
Danielle Chini
}

\section{Questionnement initial}

1 Le concept de situation s'impose comme une évidence dès qu'on parle du processus d'enseignement-apprentissage, exemple par excellence d'interaction socio-cognitive, qui, par conséquent, ne peut s'envisager que situé. Pourtant, il est difficile à cerner, soit que sa nature soit perçue comme non problématique car correspondant aux conditions environnementales matérielles de la classe, c'est-à-dire à des données objectives et définissables a priori, soit, au contraire, qu'elle soit vue comme interdépendante de l'activité qu'elle situe et que toute recherche de définition se heurte à une complexité fuyante, liée au caractère évolutif, intersubjectif et cognitif des processus en jeu. La première position est bien résumée par la définition classique de la situation comme le "cadre social et spatio-temporel dans lequel ont lieu une interaction, ou plus généralement une activité ", définition rappelée par Mondada dans le Vocabulaire des Sciences Cognitives (Houdé et al. 1998 : 114). Si de nombreux travaux semblent privilégier cette première position, les questionnements multiples auxquels ce concept donne lieu dans d'autres branches des sciences humaines, conduisent à préférer la seconde. Les questions que soulèvent ces domaines connexes à la didactique des langues, entre autres la psychologie, qu'elle soit sociale ou cognitive, et bien sûr la linguistique, qu'elle s'inscrive dans une perspective énonciative, pragmatique ou interactionnelle, gardent toute leur pertinence pour nous.

2 Il convient prioritairement de se demander s'il est justifié de limiter la portée du concept au cadre externe, circonstanciel, globalement environnemental, défini ci-dessus, ou s'il faut inclure comme relevant de la situation tous les paramètres liés aux tâches et aux sujets eux-mêmes et qui conditionnent l'activité. Cela pose le problème de la distinction faite par certains entre situation et contexte, le contexte pouvant être défini comme « un ensemble délimité de dimensions pertinentes pour l'activité en cours, alors que la 
situation serait l'ensemble des dimensions potentielles disponibles au moment de cette activité. » (Mondada 1998: 112). Étant donné le caractère socio-cognitif du processus d'apprentissage, il semble a priori difficile de maintenir, dans le cas qui nous concerne, une distinction claire entre ce qui relèverait d'une « situation » externe large d'une part et d'un « contexte » inhérent étroit d'autre part. Il suffit pour cela de penser au rôle que joue le domaine de référence lié au contenu d'apprentissage, domaine préexistant et donc « potentiellement disponible » mais aussi composante inhérente de l'activité qui se fonde sur lui mais en même temps le reconstruit. Il semble donc qu'on ne puisse échapper à une conception de la situation d'apprentissage, comme une entité complexe, présentant des strates multiples dont il conviendrait de déterminer les paramètres définitoires.

3 Cette première question en appelle deux autres. Tout d'abord, la situation doit-elle être considérée comme une entité statique et prédéterminée ou comme une construction dynamique et donc évolutive, émergeant de l'activité elle-même ? En conséquence, et pour reprendre les termes de Mondada (1998: 112-113) à propos de la nature et du rôle du contexte dans l'acte de communication, s'agit-il d'une "dimension périphérique » à l'activité d'apprentissage ou d'un élément constitutif de cette activité, qui jouerait vis-àvis d'elle et du sens qu'elle conduit à construire " un rôle fondamental et structurant "?

L'ensemble de ces questions prouve la réelle complexité du problème posé par la délimitation de ce qu'il convient de considérer comme relevant de la situation d'apprentissage. Je tenterai tout d'abord, en m'appuyant sur divers travaux portant sur les notions de situation et de contexte dans le cadre de l'interaction, d'apporter quelques éléments de réponse en explorant les caractéristiques qui me semblent les plus essentielles, à savoir la question des paramètres définitoires de la situation, celle des relations existant entre ces paramètres et enfin celle du rôle fonctionnel de la situation par rapport à l'activité, rôle qui se matérialise dans la tâche. Puis à partir d'un exemple concret, celui du déroulement d'un module de méthodologie du travail universitaire en $1 \mathrm{e}$ année de DEUG d'anglais, je présenterai les limites posées à une approche strictement externe du concept de situation d'apprentissage.

\section{Essai de définition}

\subsection{La situation comme ensemble de paramètres}

5 À première vue, parler de la situation d'une activité conduit à analyser la nature du cadre dans lequel cette activité s'insère mais aussi la manière dont elle s'insère dans ce cadre. C'est bien ce que suggère la définition du terme « situation » que donne le Petit Larousse (édition 2000), à savoir «manière dont quelque chose est placé par rapport à d'autres choses » ou encore « état de quelque chose par rapport à une conjoncture donnée dans un domaine déterminé. » Bien que très générales - il s'agit là des emplois communs du terme - ces définitions mettent clairement au centre l'idée de repérage, de localisation par rapport à un cadre de référence qui donne son sens à l'activité. Et qui dit repérage, dit repères, paramètres de repérage. Définir la situation d'apprentissage va donc passer dans un premier temps par une classification des paramètres qui permettent de localiser l'activité, en d'autres termes des différentes strates qui constituent la situation.

6 Les plus évidents sont bien sûr les paramètres institutionnels, sociaux et plus étroitement spatio-temporels, comme indiqués dans la première définition initiale que nous avons donnée. Dans le type de situation qui nous concerne ils sont assez simples à définir car ils 
sont déterminés par la nature du système éducatif, construction sociale très hiérarchisée et normée qui crée des contraintes objectives en termes de lieu, d'horaire, de contenu mais aussi de démarche et d'objectif. Ces paramètres-là, définis en particulier dans les textes officiels, sont préétablis, stables, censés connus de tous et ils échappent très largement au contrôle des participants à l'activité. Mais ils n'épuisent pas la nature de la situation d'apprentissage. S'il s'inscrit dans un cadre institutionnel et social fort, l'apprentissage n'en relève pas moins fondamentalement, comme nous l'avons dit plus haut, d'une interaction socio-cognitive, ce qui impose de prendre en compte un deuxième niveau, celui des caractéristiques intersubjectives de la situation, et d'élargir la définition à ce qui, pour certains, relève du contexte. On peut ainsi rejoindre l'analyse de Deleau, cité par Gilly, Roux et Trognon (1999: 15) et qui, à propos des interactions dialogiques, distingue trois niveaux fonctionnels du contexte, « le contexte situationnel (qui) renvoie aux conditions institutionnelles, temporelles et matérielles de la situation » et correspond donc à la première strate définie ci-dessus, puis « le contexte interactionnel, logé dans le premier, (qui) concerne les interactions proprement dites, c'est-à-dire les influences réciproques que les comportements des partenaires ont les uns sur les autres ", et enfin «le contexte interdiscursif (qui) est constitutif du contexte interactionnel mais concerne de façon spécifique le discours, c'est-à-dire les aspects langagiers des échanges par référence au contexte de la langue ».

7 Il est clair que ces deux derniers niveaux sont pertinents pour nous, même si la spécificité de l'activité d'apprentissage en modifie la nature. Ainsi dans le cas de l'enseignement collectif, le contexte interactionnel est lui-même multidimensionnel, puisqu'il faut l'appréhender aussi bien au niveau du groupe que constitue la classe, qu'au niveau des échanges en groupes réduits, constitués de manière spontanée ou provoqués par la nature de la tâche à accomplir, mais aussi au niveau de la médiation pédagogique duale entre l'enseignant-expert et l'apprenant. Chaque niveau introduit des paramètres déterminants pour définir la nature de la situation et mériterait une analyse en soi. Cela dépasserait cependant le cadre de cette intervention. Nous n'en indiquerons que les lignes générales.

8 Le premier paramètre à prendre en compte à ce niveau-là concerne les sujets, leurs caractéristiques personnelles, affectives en particulier, mais aussi ce qui est généralement désigné sous le terme d'historicité, et qu'on peut définir comme l'ensemble des expériences passées du sujet qui ont contribué à construire la manière dont il aborde son environnement, gère ses perceptions, ses relations à autrui et en construit des représentations mentales. On voit que ces paramètres, s'ils préexistent à l'activité, ne peuvent plus être considérés comme périphériques, dans la mesure où ils sont déterminants pour le déroulement de l'activité.

9 Le deuxième paramètre incontournable relève de ce qu'il convient d'appeler le contrat de communication, c'est-à-dire "l'ensemble d'attentes, règles et présupposés implicites constitutifs du métacontrat propre au contexte situationnel » qui « fixe les rapports de places, prédétermine les échanges possibles et leur impose des spécificités » (Gilly, Roux et Trognon 1999: 16) C'est, lit-on encore, «le contrat de communication inhérent au contexte situationnel qui prédétermine les buts, enjeux, contenus et formes des interactions » (1999: 20). Ce contrat est en fait ce qui, d'une part, organise le groupe en tant qu'entité et lieu de l'interaction et, d'autre part, structure la situation en tant que support de l'activité. Comme le précisent Aebischer et Oberlé (1998:1), les situations sont «caractérisées par leurs finalités, les règles qui les organisent, les conduites qui y sont 
encouragées ou interdites, les valeurs qui y sont défendues, les espaces et les temps réglés dans lesquels elles s'actualisent ».

Dans le cas de l'enseignement-apprentissage, ce contrat est bien sûr de nature pédagogique et conduit à mettre au centre la dynamique de médiation, dont il a été largement question lors du Colloque annuel 2001 du GERAS. Médiation sociale, bien sûr, qui est au coeur de l'interaction, mais aussi médiation langagière qui donne tout son sens, dans le contexte scolaire, au troisième niveau défini ci-dessus, le contexte interdiscursif. Le langage devient alors le moyen de l'apprentissage mais aussi le lieu où il se développe, ce qui justifie pleinement son inclusion dans une définition de la situation d'apprentissage. Ce lien entre contextes interactif et discursif, articulé par la présence d'un contrat fondé sur des intérêts partagés, du moins en théorie, se matérialise dans le concept de "communauté discursive", auquel Bernié consacre un article de la Revue française de pédagogie (2002). Il y rappelle la définition proposée par Bronckart (1985 : 33) pour le paramètre «lieu social» de son modèle du contexte communicatif comme une "zone de coopération dans laquelle se déroule une activité humaine spécifique à laquelle s'articule l'activité langagière ». On retrouve dans cette citation toutes les strates situationnelles précédemment répertoriées, sociale, interactionnelle et discursive. La communauté comme lieu et ciment de l'activité.

Pour résumer, on peut dire que les paramètres pertinents pour définir la situation d'apprentissage se rattachent à quatre entités :

1. l'environnement, dans un sens spatio-temporel étroit et concret, et dans un sens plus large, social et institutionnel ;

2. l'univers de référence qui recouvre en partie les paramètres socio-institutionnels par le biais en particulier du contrat de communication, mais qui surtout inclut les références à l'objet d'apprentissage et à ses règles internes ;

3. les actants ou sujets avec leurs caractéristiques multiples, affectives, historiques, cognitives ;

4. le langage dont la logique propre crée un ensemble de contraintes mais qui constitue en même temps un lieu où interaction et apprentissage peuvent s'actualiser.

La question qui se pose maintenant est de savoir comment s'articulent ces différents niveaux, ces paramètres multiples.

\subsection{La situation comme ensemble de relations}

La nature diverse des paramètres en jeu permettrait de les classer du plus objectif (comme les données institutionnelles) au plus subjectif (comme la construction discursive), ce qui reviendrait aussi à aller du plus statique, car préconstruit et échappant au contrôle des sujets et aux modifications provoquées par l'activité elle-même, au plus dynamique ou du moins fluctuant car construit par l'activité. On pourrait ainsi envisager (cf. schéma 1) un emboîtement du plus large au plus inhérent, selon le modèle des poupées russes, du social-institutionnel au discursif, du collectif à l'individuel, et procéder, pour reprendre les termes de Vion (1999: 55) à « une analyse hiérarchique [...] selon laquelle les unités d'un rang donné déterminent les unités de rang immédiatement inférieur ». 


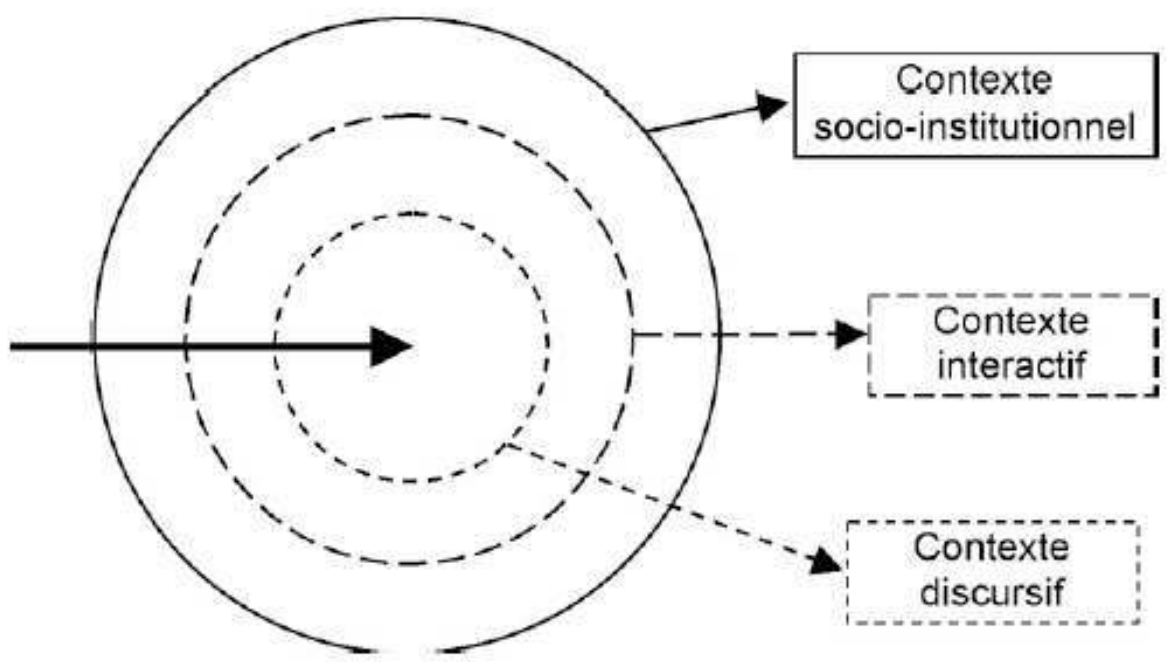
unidirectionnelle, pour reprendre les termes de Vion (1999: 56) et que si, par exemple, les modalités de l'interaction déterminent la nature des échanges discursifs, ces échanges peuvent à leur tour modifier les paramètres de l'interaction. Si on postule le même type de réciprocité à tous les niveaux, on est conduit à suivre l'analyse proposée par Vion dans son étude de l'interaction et à opter pour « une logique des relations » où les différents paramètres sont " en interrelation », ce qui conduit à « une imbrication de relations qui ne peut être représentée que spatialement", écrit Vion, et justifie pleinement l'introduction du «concept d'espace interactif. » C'est dans cette logique qu'on peut représenter la situation d'apprentissage comme un espace délimité par un noeud de relations (cf. schéma 2).

Schéma 2

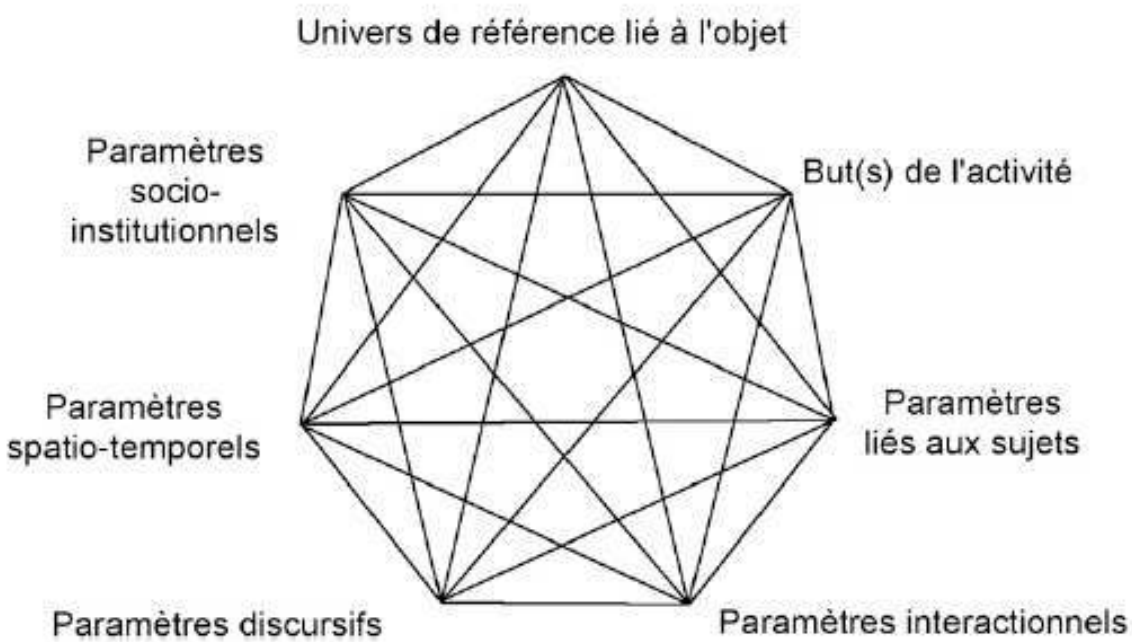

Cette représentation des paramètres définitoires de l'espace d'apprentissage conduit à redéfinir la situation non plus comme un lieu concret et prédéfini, périphérique à l'activité qu'elle situe, mais comme un espace abstrait, construit par l'interrelation entre 
des paramètres multiples, stables pour certains, fluctuants pour d'autres. Certaines de ces relations sont pour une large part prédéfinies. On peut, par exemple, penser à l'influence du cadre institutionnel sur la manière dont l'objet sera pris en compte dans les programmes officiels et donc sur les paramètres de l'objet qui seront retenus, ou encore aux contraintes créées par le cadre spatio-temporel et surtout par l'historicité des sujets sur les caractéristiques du groupe. Cependant la majorité des paramètres sont par nature fluctuants, soit de par leur caractère subjectif, soit, comme les paramètres interactionnels et discursifs, de par le lien étroit qu'ils entretiennent avec la mise en oeuvre de l'activité. Cela conduit à considérer la nature de l'espace d'apprentissage comme étant globalement évolutive, sa définition dépendant des interrelations dominantes à tel ou tel moment du processus, c'est-à-dire de la modification de certains paramètres ou même de l'introduction de paramètres nouveaux par le déroulement de l'activité. Je reprends là une opposition, établie par Michel de Certeau et reprise par Jean Caune (1999: 221) dans son ouvrage sur L'esthétique de la médiation, entre le lieu, comme «configuration de positions qui organise un ordre et implique une stabilité » et qui suppose donc une référence concrète, et l'espace qu'il présente comme "un lieu pratiqué », c'est-à-dire " animé par les mouvements humains qui s'y déploient et trouve son sens et son identité à partir des effets produits par les opérations qui s'y déroulent ».

\subsection{La situation comme composante évolutive de l'activité : le cadre de la tâche}

16 Tout cela suggère qu'on ne peut pas définir la situation sans mettre au centre l'activité qu'elle situe mais qui en même temps la construit.Ainsi c'est dans les tâches conçues et proposées par l'enseignant que se matérialise l'interrelation des différents paramètres que nous venons de considérer et donc l'espace d'apprentissage. En effet c'est à partir d'une prise en compte de ces différents paramètres que l'enseignant élabore le scénario de la tâche qui va servir de cadre à l'activité des apprenants et, dans l'idéal, créer les conditions de l'apprentissage. À partir d'une prise en compte articulée 1) des contraintes institutionnelles qui définissent les objectifs, les méthodes et les contenus, 2) des contraintes liées à la logique de l'objet, 3) de la connaissance qu'il a des apprenants et de leurs parcours antérieurs, 4) des caractéristiques de l'interaction discursive et enfin 5) de manière le plus souvent inconsciente, de ses propres habitudes et compétences en tant que médiateur. La situation d'apprentissage devient donc une composante de base de la tâche qui elle-même crée un espace où l'activité du sujet peut se développer. On en arrive ainsi à une conception totalement évolutive de la situation d'apprentissage où, pour créer les conditions d'une dynamique d'apprentissage, il convient de transformer les paramètres objectifs de nature périphérique en les intégrant comme fondement structurant de la tâche et donc à terme de l'activité.

\subsection{Conclusion}

17 En réponse aux questions initiales posées dans l'introduction, on peut donc affirmer que même si un certain nombre de paramètres situationnels sont prédéterminés et stables et peuvent sembler ne jouer qu'un rôle périphérique par rapport à l'activité d'apprentissage, on ne peut atteindre la réalité complexe de la situation d'apprentissage que si l'on s'éloigne de cette conception positiviste et qu'on l'appréhende comme une 
construction dynamique, constitutive de l'activité et évoluant avec elle. Pour autant cette définition reste globalement externe au sujet apprenant et intègre l'apprentissage comme un objectif à atteindre plus que comme une réalité. Cette définition résiste-t-elle à l'analyse d'une situation concrète, c'est ce que nous allons voir.

\section{Une situation concrète : le module de méthodologie de $1^{\mathrm{e}}$ année de DEUG d'anglais}

\subsection{Présentation de la situation mise en place}

\subsubsection{Les paramètres institutionnels}

Le module de méthodologie du travail universitaire a été introduit dans le cursus du premier semestre du DEUG d'anglais à l'Université de Pau (UPPA) à la rentrée 1999, dans le cadre de l'application de la réforme Bayrou. Il s'inscrit dans l'objectif affiché de réduction de l'échec en DEUG et se doit de familiariser les étudiants avec les spécificités des démarches universitaires. Sa mise en place devait tenir compte de paramètres institutionnels contraignants, tenant à l'horaire, à l'obligation d'une validation par examen et au coefficient affecté. Ainsi le volume horaire a été fixé à 26 heures sur 13 semaines, sans suivi au deuxième semestre, réparties en 1,5 heures de TD par semaine et par groupe. Ces TD sont complétés par une formation à la recherche documentaire assurée en CM et TP en demi-groupe par le personnel de la BU, mais articulée aux autres activités du module, en particulier par l'élaboration d'un dossier en groupe. Comme tout enseignement ce programme donne lieu à un examen terminal au mois de janvier. Ces contraintes institutionnelles se sont révélées avoir une importance primordiale sur l'efficacité de la situation mise en place. Nous y reviendrons.

\subsubsection{Les paramètres liés à l'objet et aux sujets}

19 C'est donc à partir de ces paramètres institutionnels et spatio-temporels imposés que nous avons établi une programmation hebdomadaire à partir du profil hétérogène du public et de ses besoins, estimés en fonction de la spécificité préétablie des travaux universitaires et d'une évaluation des erreurs récurrentes constatées dans notre pratique auprès des générations antérieures d'étudiants. En d'autres termes, nous avons pris en compte les paramètres objectifs liés à l'objet et les paramètres subjectifs liés en particulier à l'historicité des apprenants. Obligés de faire un choix, nous avons ciblé la structuration du discours, tout particulièrement argumentatif, en compréhension et en production, ainsi que la perception de l'implicite des documents, donnant ainsi un sens à la recherche documentaire. Ces aspects nous semblaient essentiels pour la maitrise d'activités typiquement universitaires comme la dissertation ou le commentaire de textes de littérature et de civilisation, mais aussi la prise de notes en langue étrangère ou la lecture et l'analyse d'oeuvres entières. 


\subsubsection{Définition des tâches}

Dans la logique méthodologique (cf. Chini 2001) qui a pour but de développer chez les apprenants des procédures d'action automatisées et transférables, nous nous sommes donné un certain nombre de principes pour la définition des tâches.

1. Articuler les activités méthodologiques sur le travail fait par les étudiants dans les autres composantes de leur cursus, afin de ne pas séparer la mise en place d'une démarche cognitive du contenu qu'elle était censée aider à structurer.

2. Partir systématiquement, pour construire les bases de la démarche, de l'analyse d'un travail préalable des étudiants, créant ainsi les conditions d'une confrontation des points de vue et d'une gestion raisonnée des erreurs.

3. Chercher à créer les conditions d'un réinvestissement des acquis méthodologiques comme outils structurants dans l'ensemble des composantes du cursus, au-delà du premier semestre. C'est sur cette base que les paramètres interactionnels devaient s'ancrer et c'est bien entendu dans la définition des tâches proposées que ces principes se sont matérialisés. Je ne prendrai qu'un exemple de cette démarche, celui du travail sur la prise de notes en anglais, activité particulièrement difficile pour des étudiants de 1e année. Il s'est fait à partir d'un échantillon représentatif des notes réellement prises par les étudiants lors du cours magistral de littérature de la semaine précédente, que l'enseignant avait relevées sur la base du volontariat, et photocopiées. L'objectif, à partir d'un commentaire critique en TD sur les choix de forme et de contenu différents illustrés par ces documents, était de faire prendre conscience aux étudiants de la diversité des méthodes possibles ainsi que de leurs limites ou avantages respectifs, et de les amener à interroger leur propre démarche, dans une perspective de compréhension et de mémorisation plus efficace. Ce travail était prolongé par une comparaison du contenu des notes avec 1 ) le texte initial de la préparation du cours de l'enseignant, 2) le script du cours tel que l'enseignant l'avait réellement présenté à l'oral. L'objectif était de faire prendre conscience aux étudiants du décalage entre les deux et donc des techniques rhétoriques (répétition, reformulation, explicitation, mise en valeur des articulations logiques, etc.) utilisées par l'enseignant pour faciliter leur compréhension, toutes choses qui, une fois repérées pouvaient, à notre avis, faciliter leur propre travail de structuration et de reconstruction du sens. La même démarche de confrontation et d'analyse critique et interactive de travaux initiaux a été mise en place entre autres pour l'essai, le résumé, le traitement d'une question de cours...

21 L'objectif général était d'initier des comportements (approfondissement systématique, recherche documentaire, travail en groupe...) et de les amener à percevoir la permanence de certains indices formels structurants au travers d'activités diverses, indices qui, une fois maitrisés, peuvent servir de base à une méthode de repérage systématique, rassurante et efficace pour la construction du sens. De même l'accent a été mis sur la hiérarchie des informations, la notion de mots-clefs et de niveau de sens, aspects qui prennent une importance majeure dans des études à dominante littéraire.

\subsection{Bilan de l'expérience}

\subsubsection{Constat d'échec}

La situation ainsi mise en place, fondée sur une interrelation des paramètres pertinents, du moins de notre point de vue, nous semblait à même d'éveiller le questionnement des étudiants et de les faire progresser vers l'objectif visé, c'est-à-dire une amélioration de leurs méthodes à long terme. Pourtant les résultats de cette expérience en termes 
d'apprentissage réel en fin de parcours n'ont pas été, loin de là, à la hauteur des attentes. Nos sources d'information pour l'élaboration de ce bilan ont été de plusieurs natures: tout d'abord les résultats d'un sondage écrit anonyme, effectué en fin de semestre auprès des étudiants sur ce que leur avait apporté ce travail, ensuite les résultats des examens semestriels et enfin l'évaluation subjective de l'effet du travail méthodologique effectué sur le comportement des étudiants en aval (deuxième semestre de première année et deuxième année de DEUG). Pour ce qui est de l'enquête, seuls $10 \%$ des étudiants environ ont jugé bon d'y répondre et de manière globalement négative. L'examen de janvier n'a été réussi que par un peu plus d'un quart des étudiants inscrits. Quant aux effets sur le comportement en aval il s'est révélé insignifiant, les mêmes erreurs de méthode pouvant être constatées chez ces étudiants.

Pourtant, il faut placer en contrepoint de ces résultats négatifs, le jugement très positif porté sur le processus mis en place par les spécialistes de méthodologie documentaire avec lesquels nous travaillons et qui ont déclaré: "Vous avez fait un travail très pertinent. Il faudrait que tous les départements suivent votre exemple ». D'où vient ce décalage entre les qualités apparentes du protocole fondé sur des paramètres situationnels clairement réfléchis et articulés et les résultats plus que décevants en termes d'efficacité? En d'autres termes, pourquoi une situation structurée, élaborée selon des principes didactiques apparemment cohérents, ne crée-t-elle pas les conditions d'un apprentissage positif? Mis à part les erreurs plus ou moins inévitables de mise en œuvre qui nous sont imputables, les raisons de ce décalage me semblent multiples, certaines tenant au système d'autres aux acteurs, mais l'analyse conduit surtout à revenir sur une définition strictement externe de la situation.

\subsubsection{Analyse des causes}

\subsubsection{Causes structurelles}

Tout d'abord, bien sûr, il convient de relativiser la portée de l'échec, $1 / 3$ des étudiants inscrits en début d'année abandonnant le parcours avant Noël ou ne se présentant jamais. Cette réalité nous échappe. De plus, les contraintes temporelles - il s'agit d'une formation très limitée dans le volume et la durée - sont largement contradictoires avec un apprentissage qui n'a de sens qu'à long terme, le travail sur les démarches demandant, plus encore qu'un travail sur les contenus, une remise en question d'habitudes installées qui suppose des confrontations multiples et un temps de maturation. Et puis surtout l'obligation qui nous est faite de valider ce travail par un examen spécifique va à l'encontre de l'objectif fondamental qui est de faire prendre conscience aux étudiants qu'il s'agit d'un travail transversal, qui ne prend tout son sens qu'en fonction de l'aide qu'il peut apporter au traitement des autres composantes du cursus et ce bien au-delà du premier semestre. Un contrôle continu, lourd à mettre en place, n'aurait pas réglé ce problème. Comment, par exemple, évaluer l'efficacité de la formation documentaire? Comment prendre en compte, de manière individuelle et chiffrée, le travail de recherche en groupe sur les références implicites d'un document de type civilisationnel que les étudiants devaient rendre vers la fin du semestre, quand l'essentiel est dans la démarche suivie plus que dans le résultat final ? C'est sur ce point de la validation du module que s'articule, me semble-t-il, la cause fondamentale de l'échec qui est liée à une question de représentations.

3.2.2.1 Une question de représentations : la notion de pertinence 
L'êchec tient en grande partie à la différence entre l'intention pédagogique qui sous-tend la mise en place de la situation et l'interprétation qu'en font les étudiants, c'est-à-dire la représentation, le plus souvent inconsciente, qu'ils s'en donnent. Cela conduit à introduire la notion de pertinence, chère aux spécialistes de l'interlocution. Comme le rappelle Douay (2000: 4950), en se référant à Gardiner, si «la première source d'information [...] pour la construction de la chose-signifiée [...] est fournie à l'interlocuteur par le contexte extralinguistique, ce terreau [...] que Gardiner appelle l'information d'arrière-plan ", il ne faut pas oublier que "parmi toutes les données faisant potentiellement partie de l'information d'arrière-plan, seules quel-ques-unes sont sélectionnées par l'interlocuteur » selon « le principe de l'attention sélective lié à [...] celui de la pertinence. Grâce à cette faculté d'attention sélective, tout ce qui est superflu et non indispensable à l'opérativité du discours disparaît de la conscience des interlocuteurs ou y occupe une place subalterne ». On retrouve la même idée chez Gilly, Roux et Trognon qui écrivent :

les sujets traitent de la situation à laquelle ils sont confrontés à partir de la définition qu'ils s'en donnent, c'est-à-dire des significations qu'ils lui attribuent. Ils créent leur propre représentation de la situation en fonction de leur position sociale et de leur expérience antérieure. (1999:22)

et ils ajoutent : « les partenaires peuvent donc ne pas avoir la même définition initiale de la situation ».

Il est clair que cette analyse élaborée pour l'interlocution vaut de manière plus large pour toute interaction et tout particulièrement pour la relation d'enseignement-apprentissage. La situation mise en place par l'action pédagogique fonctionne pour l'apprenant comme un contexte d'arrière-plan existant en dehors de lui et il va l'appréhender de manière sélective et l'interpréter en fonction d'une hiérarchie de pertinences qui n'est que très rarement celle de l'enseignant. C'est bien ce qu'écrit Bernié (2002) : « Tout enfant devient écolier en construisant un ensemble de représentations relatives à l'univers scolaire et ses conduites sont interprétables en bonne part comme réponse à des contextes sociaux : c'est en fonction de la signification qu'il leur attribue qu'il s'organise cognitivement à l'intérieur de la tâche et mobilise telle ou telle procédure ».

Dans le cas qui nous concerne, celui du module de méthodologie, les différences de représentations sont en fait multiples. Fondamentalement, le caractère compartimenté des enseignements, divisés en unités autonomes validées semestriellement par un examen spécifique, prévaut pour les étudiants sur le discours de transversalité, d'approfondissement et de pertinence à long terme que l'enseignant peut tenir. Et cela est d'autant plus vrai que les étudiants de première année, qui n'ont pas encore été confrontés aux difficultés et à la nouveauté des travaux universitaires, considèrent le plus souvent qu'ils sont bons en anglais et qu'ils n'ont nul besoin de cet apport méthodologique qu'ils voient comme redondant par rapport à ce qu'ils ont déjà fait au lycée. Dans le sondage cité plus haut, l'un d'eux a ainsi déclaré : «Si j'ai eu mon bac, c'est que j'ai déjà des méthodes.» En d'autres termes le paramètre pertinent pour les étudiants, et on peut le comprendre vu le poids de la sélection dans notre système, est la réussite ponctuelle à un examen, pas l'amélioration de leur démarche à long terme. C'est bien ce que montre la question qu'ils posent de manière récurrente : «Est-ce qu'on peut avoir ça à l'examen ?» Si la situation, nous l'avons dit, est définie par la finalité qu'elle vise, ce qui est déterminant, comme le rappelle Vygotski (1997: 195) à propos de la résolution des situations-problèmes, c'est «la représentation du but ». En conséquence, 
cet enseignement méthodologique à visée comportementale devient pour les étudiants un enseignement de contenu comme un autre, avec des savoirs préétablis à mémoriser, ce qui est un contresens.

En outre, leur représentation de la matière "anglais " n'est pas encore celle de spécialistes, mais celle, beaucoup moins exigeante, d'anciens lycéens pour qui il suffit d'avoir une compétence communicative moyenne pour réussir. S'ajoute à cela une conception du travail et de l'effort très différente chez les enseignants et la majorité des étudiants d'aujourd'hui. Construction à long terme qui gagne à être structurée chez les uns, effort ponctuel et ciblé pour les autres. En résumé, ce qui est déterminant pour l'efficacité du protocole mis en place c'est « le sens que les sujets attribuent à la situation, à partir d'indicateurs in situ et de leur expérience sociale antérieure " (Gilly, Roux et Trognon 1999 : 16). C'est donc ce qu'ils reconnaissent comme " contexte ", concept qu'on ne peut plus se contenter de définir comme «l'ensemble de dimensions pertinentes pour l'activité » où la pertinence semble être délimitée dans l'absolu mais, pour citer Bernié (2002), comme « l'ensemble des indices appartenant à l'univers social et reconnus comme pertinents par les enfants ${ }^{1}$ pour la construction d'une représentation des tâches ». Tout cela nous ramène à la notion d'espace d'apprentissage que nous avons définie dans la première partie et qu'il s'agit maintenant de préciser.

\section{Redéfinition de la notion de situation et d'espace d'apprentissage}

Il va de soi que l'activité d'apprentissage est fondamentalement une activité cognitive interne au sujet et qu'elle ne peut se dérouler sans implication affective et construction de sens par l'apprenant. De même la situation dite d'apprentissage ne pourra exister comme telle que si l'apprenant intériorise les paramètres qui la structurent et s'en donne une représentation positive et dynamique. Elle doit donc se définir comme l'imbrication de deux niveaux, l'un extérieur, construit par l'enseignant au travers de la tâche et commun à tout le groupe, et l'autre intérieur, c'est-à-dire individuel dans la mesure où il est construit par l'apprenant par l'interrelation des représentations qu'il se donne des paramètres reconnus par lui comme pertinents. L'espace extérieur n'est donc que potentiel et ne pourra devenir fonctionnel que si, pour chaque sujet, s'y superpose un espace cognitif intérieur. En fait, seul cet espace intérieur mérite réellement l'appellation de contexte d'apprentissage, le contexte extérieur correspondant plus justement à l'espace d'enseignement. C'est en effet à partir de cet espace intérieur et des représentations qui le construisent que l'apprenant va appréhender l'objet et construire du sens. On est là très proche de la définition que Culioli (1985: 78) donne de l'énonciation «au sens de représentation et construction de domaines sur lesquels on opère ». La situation n'a pas de sens en dehors de l'activité des sujets. En fait tout comme dans le cas de l'énonciation, on a affaire à une construction référentielle par laquelle le sujet apprenant assigne une valeur aux paramètres de la situation externe en fonction de repères internes. On est donc conduit à redéfinir la situation d'apprentissage de la même manière que la situation d'énonciation « comme un ensemble de paramètres qui forment un paquet de relations» (Culioli 1976: 93) entre références externes au sujet et références internes, c'est-à-dire entre les paramètres du cadre construit par l'enseignant à partir des données préexistantes et la valeur référentielle personnelle que l'apprenant attribue à ces paramètres. Cela peut se représenter comme dans le schéma 3. 


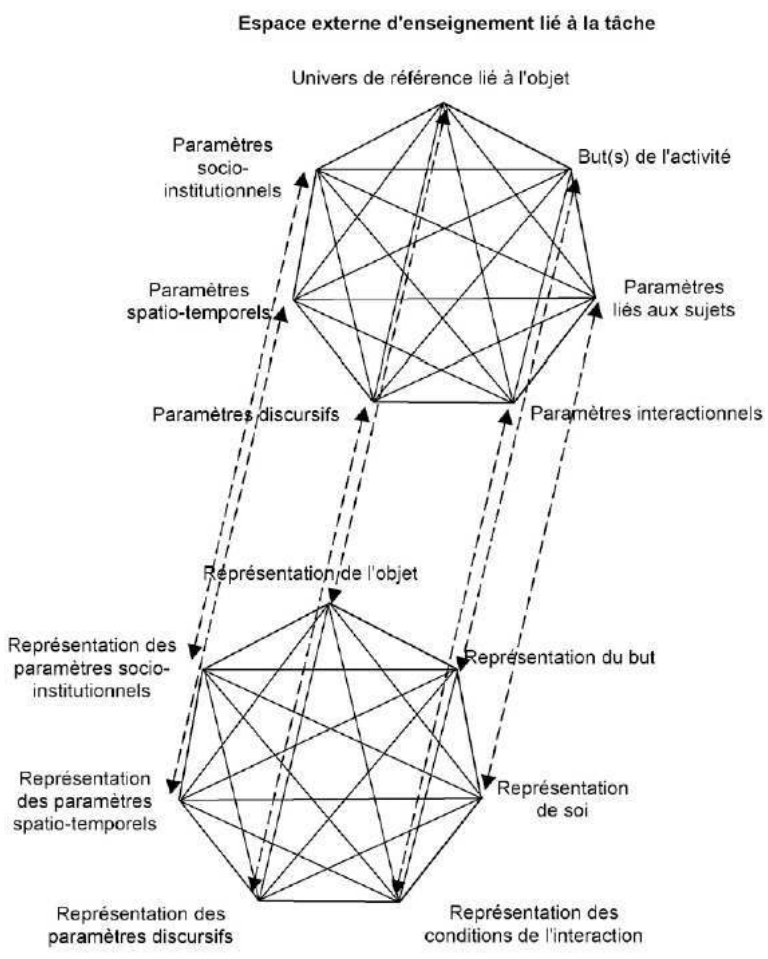

Il est évident que les deux niveaux ne sont pas homogènes, ce qui est fondamental, car c'est l'importance du décalage ou plutôt le degré de compatibilité entre les deux qui déterminera les possibilités de réussite de l'apprentissage. C'est en fait dans cet espace commun crée par la rencontre de ces deux niveaux que se situe vraiment la situation d'apprentissage et que peut intervenir la médiation enseignante. Mais si les caractéristiques du niveau externe sont pour une part importante maîtrisées de façon consciente et raisonnée par l'enseignant, les caractéristiques de l'espace interne sont, elles, largement inconscientes de la part de l'apprenant et inaccessibles à l'enseignant. Ce n'est qu'indirectement par une observation et une analyse de l'activité de l'apprenant qu'il pourra induire la nature de cet espace interne et tenter par des actions de médiation de réduire le décalage entre les deux niveaux.

\section{Conclusion}

On voit donc que le concept de situation d'apprentissage recouvre une réalité très complexe, fondée sur un ensemble de relations, intriquées et non pas emboittées, entre des paramètres externes multiples, pour certains concrets, stables et objectifs, pour d'autres abstraits ou encore subjectifs et fluctuants, mais aussi entre l'espace externe créé par l'interrelation de ces paramètres dans le cadre de la tâche et le paquet de relations nouées entre des paramètres internes, propres à chaque sujet apprenant, et émergeant des représentations qu'ils se donnent de l'espace externe. En résumé, on ne peut appréhender la complexité de ce concept que si l'on prend en compte sa composante cognitive. La situation d'apprentissage est donc bien une construction complexe et évolutive, émergeant de l'activité qu'elle contribue à construire, où un espace interne 
s'articule sur un lieu externe et où la médiation pédagogique a un rôle fondamental à jouer pour réduire les écarts entre les deux niveaux, afin de rendre cette situation opérationnelle et créer ainsi les conditions d'un apprentissage efficace. Mais comme nous l'avons vu avec l'exemple du module de méthodologie, la marge d'action peut être limitée par les paramètres externes, essentiellement institutionnels et spatio-temporels, les seuls qui échappent au contrôle des sujets, ainsi que par les représentations initiales des apprenants dont le poids peut obscurcir leur appréhension des autres paramètres et constituer des obstacles à la mise en place d'une situation dynamique réellement fonctionnelle.

\section{BIBLIOGRAPHIE}

Aebischer, Verena \& Dominique Oberlé. 1998. Le groupe en psychologie sociale. Paris : Dunod.

Bernié, Jean-Paul. 2002. «L'approche des pratiques langagières scolaires à travers la notion de ‘communauté discursive' : un apport à la didactique comparée ? ». Revue française de pédagogie 14.

Caune, Jean. 1999. Pour une éthique de la médiation : le sens des pratiques culturelles. Grenoble :

Presses Universitaires de Grenoble.

Chini, Danielle. 2001. «L'objectif méthodologique : construction d'une mémoire procédurale?» Journée d'étude du Groupe d'étude en pyscholinguistique et didactique (GEPED). Université de Pau et des Pays de l'Adour. À paraître dans les actes.

Culioli, Antoine. 1976. Recherche en Linguistique : Théorie des opérations énonciatives.Transcription du séminaire de DEA 1975-1976, Département de Recherches Linguistiques, Université Paris VII, ronoétypé.

Culioli, Antoine. 1985. Notes du séminaire de DEA 1983-1984. Département de Recherches Linguistiques, Université Paris VII, ronéotypé.

Douay, Catherine. 2000. Éléments pour une théorie de l'interlocution : un autre regard sur la grammaire anglaise. Rennes : Presses Universitaires de Rennes.

Gilly, Michel, Jean-Paul Roux \& Alain Trognon (dir.). 1999. Apprendre dans l'interaction. Nancy : Presses Universitaires de Nancy et Presses Universitaires de Provence.

Houdé, Olivier et al. 1998. Vocabulaire de sciences cognitives. Paris : PUF.

Vion, Robert. 1999. « Linguistique et communication verbale. » In Gilly, Roux \& Trognon (dir.), Apprendre dans l'interaction. Nancy : Presses Universitaires de Nancy et Presses Universitaires de Provence, 41-64.

Vygotski, Lev. 1997. Pensée et langage. $3^{\mathrm{e}}$ édition. Traduction de Françoise Sève. Paris : La Dispute.

\section{NOTES}

1. Bernié se situant dans le cadre de l'école primaire parle d'enfants pas d'étudiants. 


\section{RÉSUMÉS}

À première vue, la situation d'apprentissage s'impose comme un lieu d'interaction sociale où les participants s'inscrivent dans une relation de médiation. De nature complexe, elle est constituée de strates interdépendantes, institutionnelle aussi bien que groupale ou discursive, ou encore liée au contexte de la tâche. Cependant l'analyse de ces strates ne saurait suffire à définir la nature profonde de la situation d'apprentissage, dans la mesure où elles ne déterminent qu'un lieu extérieur au sujet, dont les paramètres sont définis a priori. Or il ne peut y avoir situation d'apprentissage à proprement parler que s'il y a construction d'un espace cognitif interne au sujet, sous l'effet de la mise en relation dynamique et de l'intériorisation des paramètres prédéfinis. En s'appuyant sur sa pratique à l'université, l'auteur montrera les difficultés que pose la mise en œuvre de cette dynamique et de quelle manière le caractère contraignant de certains paramètres, institutionnels en particulier, peut bloquer le processus d'intériorisation. Ce bilan conduit à se poser la question des conditions d'une médiation efficace à l'université, colonne vertébrale d'une situation d'apprentissage opérationnelle.

A learning situation can be defined as an environment set up for the development of a specific form of mediated social interaction. Its complex nature derives from the interconnection of multiple frames, as different as institutional constraints and task-induced contexts, or the rules of discourse or of group organisation. Yet assessing those different frames is not enough to account for the real nature of a learning situation in so far as most of them are set according to pre-existing parameters and therefore defined with no control on the part of the learner. Yet a learning situation can only be considered as such once the learner has internalized those various parameters and frames and built a mental representation of them, thus delimiting a new inner space whose subjective and cognitive nature is decisive in the dynamic of the learning process. Practical evidence abounds that the reality of the learning process relies on the capacity to create the conditions for that problematic transition - from a predefined collective environment to a multiplicity of individual mental spaces. That is where difficulties lie when trying to set up an efficient mediation and a successful learning situation.

\section{INDEX}

Keywords : cognitive construct, interaction, mental image, multidimensional space, parameter

Mots-clés : construction, espace multidimensionnel, interaction, paramètre, représentation

\section{AUTEUR}

\section{DANIELLE CHIN}

Danielle Chini est titulaire depuis 1996 d'une thèse de doctorat nouveau régime intitulée « La problématique linguistique de l'enseignement de l'anglais dans le secondaire ». Elle est maître de conférences à l'Université de Pau et des Pays de l'Adour, où elle assure des enseignements de linguistique et de didactique de l'anglais ainsi que de psycholinguistique. Elle dirige depuis le 
printemps 2000 le G.E.P.E.D. (Groupe d'étude en psycholinguistique et didactique), groupe de recherche créé par le professeur Danielle Bailly et initialement rattaché à l'Université Paris 7Denis Diderot. Ses domaines de recherche privilégiés sont les relations entre la didactique de l'anglais d'une part et la linguistique et la psycholinguistique d'autre part, ainsi que toutes les questions qui se rattachent à la problématique de la conceptualisation dans l'apprentissage des langues.danielle.chini@univ-pau.fr 